\title{
A RIG-I 2CARD-MAVS200 Chimeric Protein Reconstitutes IFN- $\beta$ Induction and Antiviral Response in Models Deficient in Type I IFN Response
}

\author{
Estanislao Nistal-Villán ${ }^{a, d}$ Estefanía Rodríguez-García a, d Marianna Di Scala a, d \\ Roberto Ferrero-Laborda ${ }^{a} d$ Cristina Olagüe ${ }^{a, d}$ África Vales ${ }^{a, d}$ \\ Beatriz Carte-Abad ${ }^{a, d}$ Irene Crespo ${ }^{e}$ Adolfo García-Sastre ${ }^{f-h}$ \\ Jesús Prieto a, b, d Esther Larrea a, c, d Gloria González-Aseguinolazaa,d
}

${ }^{a}$ Centro de Investigación Médica Aplicada, Gene Therapy and Regulation of Gene Expression Program, ${ }^{b}$ Clínica Universidad de Navarra, CIBERehd and 'Instituto de Salud Tropical, University of Navarra, and ' IdiSNA Navarra Institute for Health Research, Pamplona, and ${ }^{\mathrm{e}}$ Institute of Biomedicine (IBIOMED), CIBERehd, University of León, León, Spain; ${ }^{\mathrm{f}}$ Department of Microbiology, ${ }^{9}$ Global Health and Emerging Pathogens Institute, and hepartment of Medicine, Icahn School of Medicine at Mount Sinai, New York, N.Y., USA

\section{Key Words}

Immunostimulation · Interferon- $\beta$ - RIG-I · MAVS .

Adeno-associated virus · Viral antagonist

\begin{abstract}
RIG-I-like receptors (RLRs) are cellular sensor proteins that detect certain RNA species produced during viral infections. RLRs activate a signaling cascade that results in the production of IFN- $\beta$ as well as several other cytokines with antiviral and proinflammatory activities. We explored the potential of different constructs based on RLRs to induce the IFN- $\beta$ pathway and create an antiviral state in type I IFN-unresponsive models. A chimeric construct composed of RIG-I 2CARD and the first 200 amino acids of MAVS (2CARD-MAVS200) showed an enhanced ability to induce IFN- $\beta$ when compared to other stimulatory constructs. Furthermore, this human chimeric construct showed a superior ability to activate IFN- $\beta$ expression in cells from various species. This construct was found to overcome the restrictions of blocking IFN- $\beta$ induction or signaling by a number of viral IFN-antagonist proteins. Ad-
\end{abstract}

ditionally, the antiviral activity of this chimera was demonstrated in influenza virus and HBV infection mouse models using adeno-associated virus (AAV) vectors as a delivery vehicle. We propose that AAV vectors expressing 2CARDMAVS200 chimeric protein can reconstitute IFN- $\beta$ induction and recover a partial antiviral state in different models that do not respond to recombinant IFN- $\beta$ treatment.

(c) 2015 S. Karger AG, Basel

\section{Introduction}

Cellular innate immunity is the first line of defense against infections. In higher vertebrates, innate immunity activation results in a coordinated response that relies on a rapid detection of pathogens by pattern recognition receptors (PRRs). PRRs recognize pathogen-specific signatures called pathogen-associated molecular patterns (PAMPs) [1]. PRR activation initiates a direct response against infection through the stimulation of specific signaling pathways, such as those leading to IFN- $\beta$ induc-

\section{KARGER 125}

2015 S. Karger AG, Base

$1662-811 X / 15 / 0075-0466 \$ 39.50 / 0$

E-Mail karger@karger.com

www.karger.com/jin
Dr. Estanislao Nistal-Villán and Dr. Gloria González-Aseguinolaza

Centro de Investigación Médica Aplicada (CIMA)

Gene Therapy and Regulation of Gene Expression Program, University of Navarra

Ave. Pío XII no 55, ES-31008 Pamplona (Spain)

E-Mail enistal@unav.es and ggasegui@unav.es 
tion and the activation of the adaptive arm of the immune response [2]. As a result, the host acquires a refractory state against the infection [3].

Cytosolic PRRs such as the RIG-I-like receptor (RLR) family play a critical role in the activation of cellular antiviral innate immunity. The RLR family comprises three proteins: retinoic acid inducible gene-I (RIG-I), melanoma differentiation-associated protein 5 (MDA5) and the laboratory of genetics and physiology protein 2 (LGP2). RIG-I, MDA5 and LGP2 can recognize and become activated by RNA species generated during virus replication. RIG-I and MDA5 contain an amino-terminal region composed of two caspase activation and recruitment domains (2CARD), a central RNA helicase domain and a carboxy-terminal regulatory domain [4]. In the absence of PAMP stimulation, RIG-I remains in an inactive closed conformation. Upon binding to the appropriate RNA, a conformational change occurs exposing the 2CARD domain which is dephosphorylated and released from its interaction with the RNA helicase. The 2CARD domain is then free to bind polyubiquitin chains which promote its association with the cytosolic CARD domain of mitochondrial antiviral signaling protein (MAVS) [4]. This interaction determines the stimulation of the IFN- $\beta$ induction pathway [5].

Downstream of MAVS, the signaling pathway splits into three branches. TBK1 and/or IKKe can phosphorylate and activate interferon regulatory factor 3 (IRF3) and/or IRF7 [6]. These transcription factors stimulate IFN- $\beta$ gene transcription as well as several other genes by binding to IRF-binding elements (IRF-E) [7]. Examples of genes with IRF-E elements include IFN-stimulated genes (ISGs) ISG15, ISG54 or ISG56 [8]. MAVS signaling also stimulates the IKKa $\beta \gamma$ kinase complex, resulting in the activation of $\mathrm{p} 50 / \mathrm{p} 65$ nuclear factor $\kappa \mathrm{B}(\mathrm{NF}-\kappa \mathrm{B})$ which is essential for IFN- $\beta$ production and other NF- $\kappa \mathrm{B}-$ dependent genes such as CXCL10/IP10 [9]. Finally, the mitogen-activated protein kinase pathway is triggered and stimulates the activator protein 1 (AP1), also involved in IFNB1 gene transcription.

Once transcribed and translated, IFN- $\beta$ is secreted and binds type I IFN receptor. The type I IFN signaling cascade is triggered by activation of the Jak 1 and Tyk 2 kinases and phosphorylation of STAT1 and STAT2. These transcription factors recruit IRF9 and form a complex named ISGF3, which translocates to the nucleus and binds DNA elements called IFN-stimulated response elements or ISRE [10] on the promoter of ISGs.

The overall level of virus replication in a cell is determined by the amount of cellular components available for its replication and the activation status of the innate immune system involved in the antiviral response. To counteract the immune response, viruses have evolved mechanisms that block antiviral signaling cascades at various points [11]. Increasing the relative amounts of antiviral proteins commonly targeted by viral antagonists is likely to result in an enhanced antiviral response and subsequent repression of virus replication [12].

To date, overexpression of chimeras containing key domains that activate IFN- $\beta$ induction has not been explored. This approach may be especially effective if the designed chimeric protein is more potent than its wildtype (WT) protein counterparts.

In this study, we determine the antiviral potential of RIG-I2CARD fused to the CARD-PRR region(MAVS200) of MAVS and test its relative antiviral activity in vitro and in vivo. We also explore the antiviral effect of this construct using adeno-associated virus (AAV) vectors in a mouse model of acute influenza virus infection and in a model of chronic infection using HBV transgenic mice.

\section{Materials and Methods}

\section{Cells}

HEK293, 293T, BHK and L929 cells were obtained from ATCC. Statens Seruminstitut rabbit cornea (SIRC) cells used in this study have been described previously [13]. The A549 cells were a kind gift from Dr. Heinrich Hoffmann (Microbiology Department, Icahn School of Medicine at Mount Sinai), the hamster pancreatic carcinoma-derived HaP-T1 cells from Dr. Rubén Hernández-Alcoceba (CIMA, Universidad de Navarra), the woodchuck hepatoma WCH-17 cells from Dr. Michael Roggendorf (Center for Biomedical Biotechnology-Universitat Duisburg Essen) and Huh7.0 cells expressing HCV genome were generated as described previously [14]. STAT1 knockout (KO) and corresponding WT mouse embryo fibroblasts (MEFs) as well as WT and KO IPS1 MEFs have been described previously $[15,16]$. Cells were cultured in Dulbecco's modified Eagle's medium supplemented with $10 \%$ heat-inactivated FBS, $2 \mathrm{mM}$ L-glutamine, $50 \mu \mathrm{g} / \mathrm{ml}$ penicillin/streptomycin (all culture reagents from Invitrogen). All the cells were grown at $37^{\circ} \mathrm{C}$ in a $5 \% \mathrm{CO}_{2}$ incubator. Stable 293 Simian virus 5 (SV5) V cells were obtained by transfecting cells with pdlPIV5 V plasmid, kindly donated by Dr. Rick Randall (Biomedical Sciences Research Complex, University of Saint Andrews). Transduced stable cells were selected by maintaining them in Dulbecco's modified Eagle's medium complete media containing $2 \mu \mathrm{g} / \mathrm{ml}$ of puromycin. Resistant cells were maintained in the same media but with $0.25 \mu \mathrm{g} / \mathrm{ml}$ of puromycin.

\section{Reporter Assays}

IFN- $\beta$ induction in HEK293T cells was performed as described before using a pGL4.17 mIFN- $\beta$ firefly (FF) luciferase reporter plasmid [17], a kind gift from Dr. Juan Ayllon, (Department of Microbiology, Icahn School of Medicine at Mount Sinai). Reporter assay 
analysis by flow cytometry was performed in the same way but using pIFN- $\beta$-GFP [18] and pEGFP-C1-iRFP (Addgene) fluorescence reporter plasmids. Cells were analyzed $18 \mathrm{~h}$ after transfection.

\section{Viruses}

Sendai virus Cantell strain $(\mathrm{SeV})$ was grown by low-dilution passage $(1: 1,000)$ in 10 -day-old embryonated chicken eggs for 2 days. Allantoid fluid was cleared by centrifugation at $10,000 \mathrm{~g}$ for $15 \mathrm{~min}$, aliquoted and quick frozen in dry ice and stored at $-80^{\circ} \mathrm{C}$. Relative quantification of viral particles was measured with the hemagglutination assay using $0.5 \%$ fresh chicken red blood cells in PBS. Virus stock was made with eggs containing 14 wells of hemagglutination. Cells were infected with approximately 30,000 hemagglutination assay units.

Stock of influenza A/PR/8/34 was grown in MSCK-NS1 cells (a kind gift from Dr. Luis Martínez-Sobrido, Department of Microbiology and Immunology, University of Rochester Medical Center). Two days after inoculation, cell supernatants were collected, cleared by centrifugation at $10,000 \mathrm{~g}$ for $15 \mathrm{~s}$, aliquoted and frozen in dry ice. Virus titers were determined by the plaque assay [19].

VSV(M51R)-GFP was grown in BHK cells. Two days later, cell supernatants were collected, cleared by centrifugation at $10,000 \mathrm{~g}$ for $15 \mathrm{~s}$, aliquoted and frozen in dry ice. Virus stock was titrated with the Reed-Muench method [20] in Vero cells.

Recombinant rNDV-F3aa-GFP LaSota virus was rescued in our laboratory as previously described [21]. Virus was grown in 10-day-old embryonated chicken eggs for 2 days. Allantoid fluid was cleared by centrifugation at $10,000 \mathrm{~g}$ for $15 \mathrm{~s}$, aliquoted and quick-frozen in dry ice and stored at $-80^{\circ} \mathrm{C}$. Virus was titrated by immunofluorescence using polyclonal sera anti-NDV-LaSota (a kind gift from Dr. Qinshan Gao, Department of Microbiology, Icahn School of Medicine at Mount Sinai).

\section{Immunofluorescence}

A549 cells were transfected in suspension with $1 \mu \mathrm{g}$ of the indicated plasmids using Lipofectamine 2000 and seeded (BD Falcon Culture Slides). Cells were washed $18 \mathrm{~h}$ later, and fixed with $4 \%$ paraformaldehyde in PBS. After $20 \mathrm{~min}$, cells were washed 3 times with PBS for a total of $30 \mathrm{~min}$ and then permeabilized with $0.25 \%$ of Triton X-100 in PBS for 60 min. Next, cells were blocked with $10 \%$ FBS in PBS at room temperature for $30 \mathrm{~min}$. They were then incubated with a primary antibody (M2 FLAG monoclonal antibody-Sigma) diluted 1:1,000 in blocking buffer or rabbit polyclonal anti-COX IV-mitochondrial loading control (Abcam). Cells were washed 3 times with $0.1 \%$ Tween 20 in PBS for a total of $20 \mathrm{~min}$. Next, cells were incubated for $2 \mathrm{~h}$ with the Alexa Fluor 555 secondary anti-mouse and Alexa Fluor 488 anti-rabbit antibodies (Life Technologies) diluted 1:1,000 in PBS. Finally, they were washed 3 times with $0.1 \%$ Triton X-100 in PBS for a total of $30 \mathrm{~min}$. Cells were then mounted in 1:1 glycerol:PBS containing TO-PRO-3 (Life Technologies) following the manufacturer's instructions. Images were taken using a Zeiss Axioplan 2 imaging microscope.

Gene Expression Analysis

Total RNA was isolated with Trizol reagent (Invitrogen) and reverse-transcribed using random primers and Moloney murine leukemia virus (M-MLV) reverse transcriptase (Invitrogen) according to the manufacturer's instructions.

For quantitative PCR, cDNA was amplified using iQ SYBR ${ }^{\circledR}$ Green Supermix according to the manufacturer's instructions in a
C1000 thermal cycler (Biorad). The sequence of the primers used is shown in the online supplementary table 1 (for all online suppl. material, see www.karger.com/doi/10.1159/000375262).

\section{Plasmid Construction}

Human flag-tagged RIG-I 2CARD and human flag-tagged MAVS were generated by PCR amplification of specific sequences using as a template plasmids bearing RIG-I or MAVS cDNAs and cloning into pCAGGS plasmid previously digested with EcoRI and NheI. The primers used are listed in online supplementary table 1: S-EcoRI-Flag-hCARD211 +1 and A-STOP-NheI-h2CARD for 2CARD; S-EcoRI-Flag-hMAVS and A-STOP-NheI-hMAVS for full length MAVS and S-EcoRI-Flag-hMAVS and A-STOP-NheMAVS200 for MAVS200. The human fusion protein flag-tagged 2CARD-MAVS200 was generated by overlapping PCR using S-EcoRI-Flag-hCARD211 +1 and A-2hCARD-KpnI-hMAVS to amplify 2CARD and S-2hCARD-KpnI-hMAVS and A-STOP-NheMAVS200 to amplify MAVS200. pGAGGS human flag-tagged RIGI construction has been described previously [22]. 2CARD, MAVS and 2CARD-MAVS200 constructs were subcloned into AAV-IFN plasmid by replacing wIFNa5 [23] with the corresponding inserts.

\section{HBcAg Quantification}

To analyze $\mathrm{HBcAg}$ expression, digital images were acquired with an AxioImager.MI microscope (Zeiss, 20 Germany) using an in-house Metamorph macro (Molecular Devices, USA) that allows capture of randomly located images at $\times 20$ magnification (PlanNeofluar objective, $0.50 \mathrm{na}$ ) with automatic focus, white balance and shadow correction on every acquired image. All images were stored in uncompressed 24-bit color TIFF format. Images were automatically analyzed using a plugin developed by Fiji, ImageJ [24].

\section{Bioassay to Determine Antiviral Activity}

293 cells were transfected in suspension with $0.5 \mu \mathrm{g}$ of plasmids of the corresponding constructs. Twenty-four hours later, supernatants were collected and diluted in 1:2 dilution series in cell media. Dilutions were transferred onto 96-well plates of Vero cells and incubated for $24 \mathrm{~h}$. Media were removed and replaced with $50 \mu \mathrm{l}$ of fresh media containing the appropriate dose of NDVF3aa-GFP for a MOI of 1 . Virus replication was monitored $18 \mathrm{~h}$ later under a fluorescence microscope where the images were collected. GFP intensity was quantified using ImageJ software.

\section{Animals and Treatment}

HBV transgenic mouse lineage 1.3.32 (inbred C57BL/6, H-2 ${ }^{\mathrm{b}}$ ) was kindly provided by Professor Frank Chisari (The Scripps Research Institute, La Jolla, Calif., USA). HBV transgenic mice encode a 1.3-overlength copy of the HBV genome (serotype ayw), replicate $\mathrm{HBV}$ at high levels in the liver and express all of the HBV antigens [25]. HBV transgenic mice were matched for age (10 weeks), sex (female) and serum HBV DNA analysis and hepatitis B surface antigen (HBsAg) levels, as determined by an enzyme-linked immunosorbent assay (ELISA, Bioelisa HBsAg 3.0, Biokit). Mice were bred and maintained under pathogen-free conditions at the animal facility of the University of Navarra (protocol No. 149/10).

Mice were injected intravenously with the abovementioned AAV viruses at doses of $5 \times 10^{9}, 1 \times 10^{10}$ and $5 \times 10^{10}$ viral genomes $(\mathrm{vg}) /$ mouse. Blood was collected from the retro-orbital plexus, and serum samples were obtained by the recovery of supernatant after the centrifugation of total blood. 


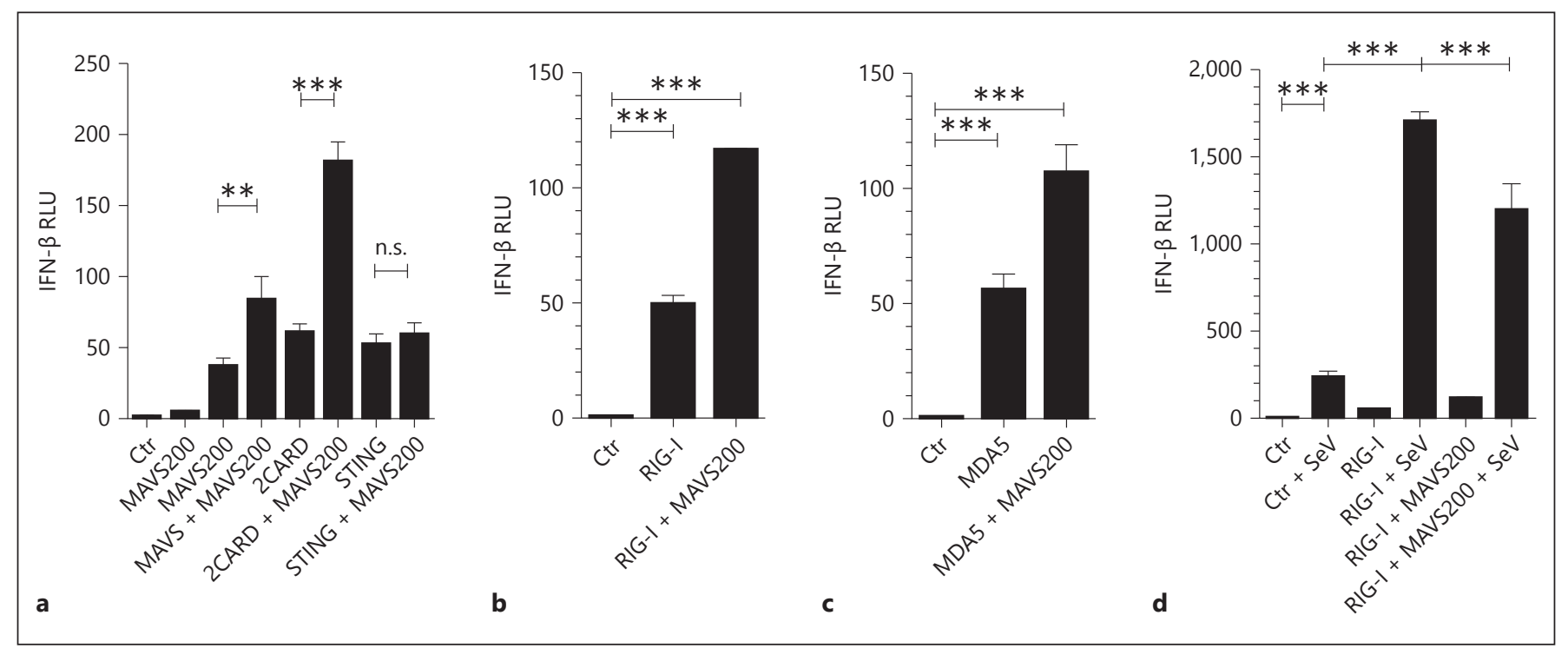

Fig. 1. MAVS construct containing the first 200 amino acids of MAVS can enhance RLR stimulation of IFN- $\beta$. a IFN- $\beta$ reporter assay of $293 \mathrm{~T}$ cells transfected with reporter plasmids together with plasmids that express MAVS, RIG-I 2CARD or STING with or without MAVS200. IFN- $\beta$ reporter assay of 293 T cells transfected with corresponding reporter plasmids together with $1 \mu \mathrm{g}$ of full-length RIG-I (b) or full-length MDA (c) together with an emp-

C57BL/ 6 female mice, aged 6 weeks, were purchased from Harlan Laboratories (Barcelona, Spain). They received a single intranasal administration of the corresponding AAV viruses at a dose of $10^{11} \mathrm{vg} /$ mouse. Three days later, they were anesthetized and infected intranasally with the different amounts of influenza A/ $\mathrm{PR} / 8 / 34$ virus (in $50-\mu$ l volumes) indicated below. For the determination of lung virus titers, the mice were euthanized either on day 3 or day 6 after infection. Lungs were homogenized and resuspended in $1 \mathrm{ml}$ of sterile PBS, and the titers were evaluated on MDCK-NS1 cells in the presence of $1.5 \mu \mathrm{g}$ of TPCK-treated trypsin $/ \mathrm{ml}$.

For all procedures, animals were anesthetized by intraperitoneal injection of a mixture of xylacine (Rompun 2\%, Bayer) and ketamine (Imalgene 500, Merial) 1:9 v/v. The experimental design was approved by the Ethical Committee for Animal Testing of the University of Navarra.

\section{FACS Analysis}

For flow-cytometry analysis, the 8-color BD FACS Canto II equipped with 488-nm and 633-nm diodes or the 4-color FACS Calibur with two lasers (argon-ion, 488-nm and 635-nm diodes) were used. Cells were analyzed with FlowJo and DIVA software.

\section{Statistical Analysis}

Statistical analysis was performed using PRISM version 5.0 (GraphPad). Data are presented as mean \pm SD. Comparisons between 2 groups were made using a 2-tailed unpaired t test. Multiple groups were compared using ANOVA followed by a Bonferroni post-test. Statistical significance was assigned to $\mathrm{p}<0.001, \mathrm{p}<0.01$ or $\mathrm{p}<0.05$. Mouse survival analysis was performed using the $\mathrm{Ge}-$ han-Breslow-Wilcoxon test.

A RIG-I 2CARD-MAVS200 Chimera

Restores Type I IFN Response ty plasmid or a plasmid that expresses MAVS200. d IFN- $\beta$ reporter assay of 293T cells transfected with corresponding reporter plasmids. In addition, cells were either transfected with full-length RIG-I or an empty plasmid and MAVS 200. Twenty-four hours later, cells were infected with $\mathrm{SeV}$ and analyzed $18 \mathrm{~h}$ later. $\mathrm{Ctr}=$ Control; n.s. $=$ not significant. ${ }^{* *} \mathrm{p}<0.01,{ }^{* * *} \mathrm{p}<0.001$.

\section{Results}

\section{Coexpression of MAVS200 with 2CARD Potentiates \\ IFN- $\beta$ Induction}

The interaction between the RIG-I 2CARD domain with the CARD proline-rich domain (CARD-PRD) region of MAVS (MAVS200) has been previously demonstrated [22]. In our studies, we observed that coexpression of these two elements had an enhanced ability to induce the activation of the IFN- $\beta$ pathway in comparison to the expression of each individual element. To characterize this effect, we tested the ability of MAVS200 to synergize with other MAVS interacting proteins to induce IFN- $\beta$ in $293 \mathrm{~T}$ cells. As expected, overexpression of MAVS200 alone failed to stimulate IFN- $\beta$ induction. Coexpression of MAVS200 together with full-length MAVS had a small but significant effect on MAVS ability to induce IFN- $\beta$ (fig. 1a). However, coexpression of MAVS200 and RIG-I 2CARD significantly increased the ability of 2CARD to induce the pathway. MAVS200, however, was not able to modify STING ability to induce IFN- $\beta$. Next, we analyzed whether MAVS200 could also cooperate in the ability of RIG-I and MDA5 to induce IFN- $\beta$. As shown, MAVS200 had a significant cooperative effect on both RIG-I (fig. 1b) and MDA5 (fig. 1c). 


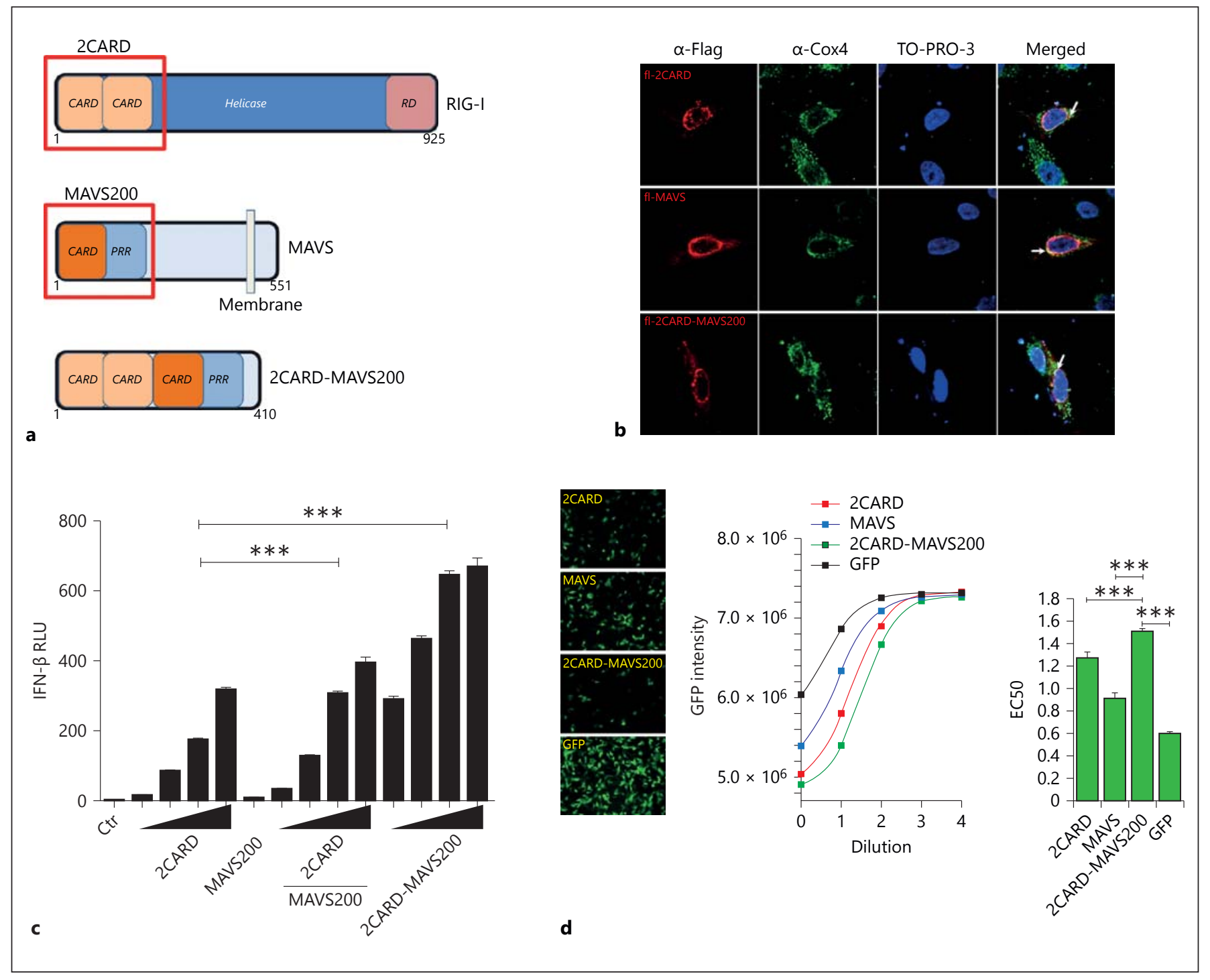

Fig. 2. Chimeric 2CARD-MAVS200 protein has an enhanced ability to activate IFN- $\beta$ induction compared to RIG-I 2CARD or MAVS. a Schematic representation of the different proteins and domains used for the construction of the 2CARD-MAVS200 chimera. b Immunofluorescence showing cellular localization of RIG-I 2CARD, MAVS or 2CARD-MAVS200 (red). Mitochondrial localization is shown by detection of mitochondrial marker Cox4 (green). Nuclear localization was detected by TO-PRO-3 (blue). c IFN- $\beta$ reporter assay in $293 \mathrm{~T}$ cells transfected with increasing amounts of RIG-I 2CARD domain or 2CARD together

As RIG-I can act as a PRR to detect SeV infection [26], we tested the ability of MAVS200 to cooperate with RIG-I in the activation of IFN- $\beta$ induced by $\mathrm{SeV}$ infection. In this context, coexpression of RIG-I and MAVS200 did not induce IFN- $\beta$; in fact, its expression was slightly diminished (fig. 1d). with MAVS200. In parallel, cells were transfected with the chimeric construct 2CARD-MAVS200. d IFN bioassay in 293 cells transfected with $1 \mu \mathrm{g}$ of 2CARD-, MAVS- or 2CARD-expressing plasmids. Twenty-four hours after transfection, cell supernatant dilutions were transferred to Vero cells, $24 \mathrm{~h}$ after supernatant transfer, cells were infected with a MOI of 1 of NDV-GFP and $18 \mathrm{~h}$ later, GFP fluorescence of infected cells was analyzed. EC50 represents an effective concentration of supernatant dilution that is able to block NDV-GFP fluorescence by $50 \%{ }^{* * *} \mathrm{p}<0.001$.

\section{Construction and Characterization of a}

\section{CARD-MAVS200 Fusion Protein}

Based on the ability of MAVS200 to enhance 2CARDmediated IFN- $\beta$ induction, we decided to create a fusion protein bearing RIG-I 2CARD and the first 200 amino acids of MAVS protein (fig. 2a). Protein expression levels 


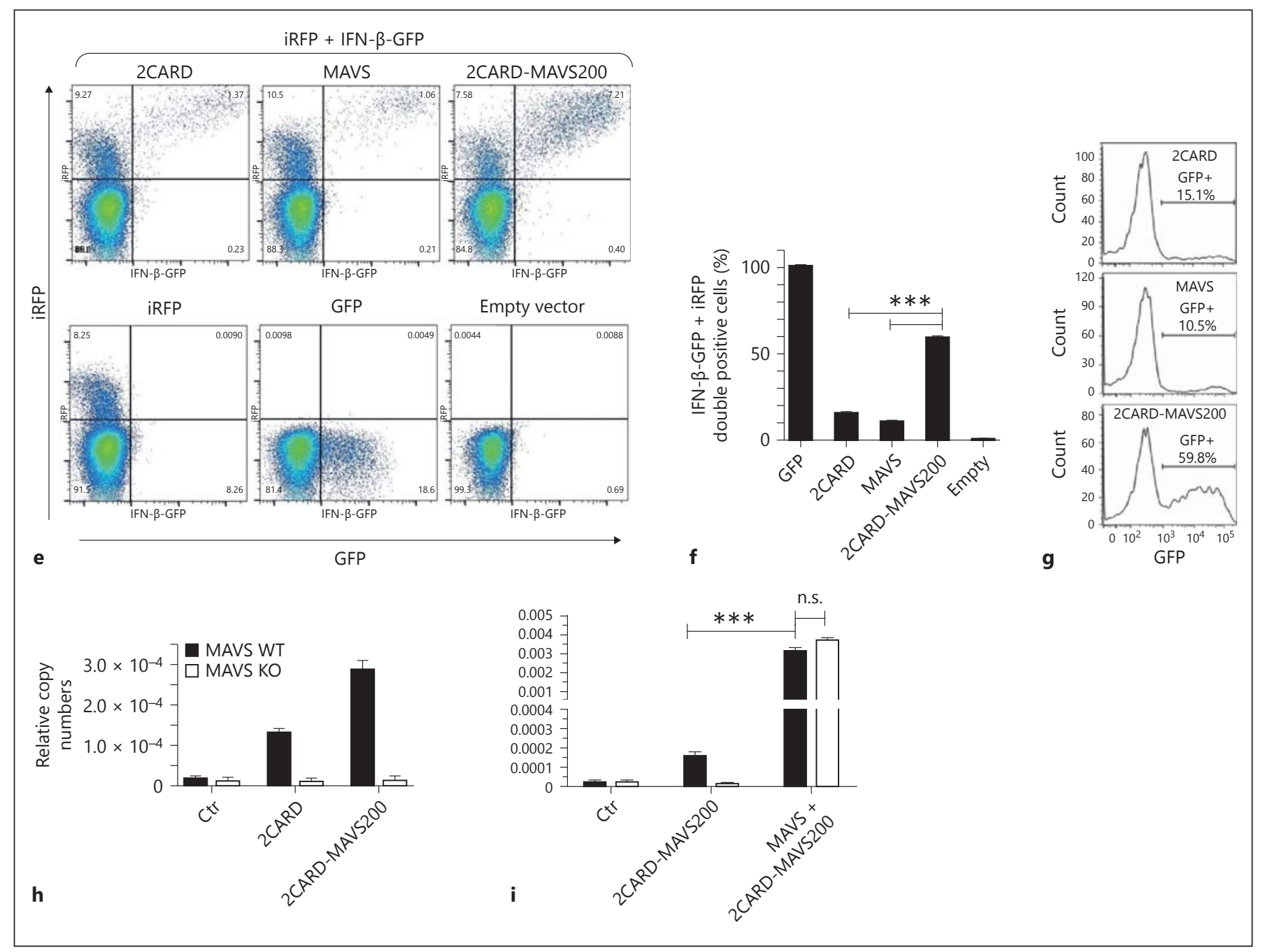

Fig. 2. Chimeric 2 CARD-MAVS200 protein has an enhanced ability to activate IFN- $\beta$ induction compared to RIG-I 2CARD or MAVS. e-g Analysis of the population of cells that induce IFN- $\beta$ by flow cytometry. Cells were transfected with a mix of an IFN- $\beta$ GFP reporter plasmid together with a CMV dependent-iRFP reporter plasmid. In addition, cells were cotransfected with $30 \mathrm{ng}$ of plasmids expressing 2CARD, MAVS or 2CARD-MAVS200. Control group cells received only the plasmid expressing iRFP, GFP or

in 293T cells transfected with a plasmid containing 2CARD-MAVS200 were comparable to those obtained after transfection of 2CARD or MAVS (data not shown). Next, we analyzed the localization of the three different proteins inside the cell by immunofluorescence. All the proteins showed partial mitochondrial localization in perinuclear areas, MAVS and 2CARD-MAVS200 showed larger areas of intense colocalization with a mitochondrial marker (fig. $2 b$, pointed with white arrows) than 2CARD. empty plasmids. GFP and iRFP were detected in FL1 and PE-Cy7, respectively. e Dot-plot representation of cells. $\mathbf{f}$ Bar graph representing the percentage of IFN- $\beta$-GFP-positive cells within the iRFP-positive populations. $g$ Histogram of GFP cells. Percentage of GFP-positive cell populations. $\mathbf{h}, \mathbf{i}$ Quantification of endogenous IFNB1 mRNA expression by qRT-PCR in WT or KO IPS MEFs transfected with the indicated plasmids. Ctr = Control; n.s. $=$ not significant. ${ }^{* * *} \mathrm{p}<0.001$.

We next compared the ability of the chimeric protein to stimulate IFN- $\beta$ production with that of MAVS200 or 2 CARD. We performed an IFN- $\beta$ reporter assay by transfecting 293T cells with increasing amounts of plasmids bearing 2CARD alone or 2CARD coexpressed with MAVS200 or with increasing amounts of 2CARD-MAVS 200 (fig. 2c). The fusion protein 2CARD-MAVS200 enhanced IFN- $\beta$ induction at all concentrations tested in comparison to the coexpression of 2CARD and MAVS200 separately. Additional combinations of MAVS200 and 
2CARD with the different construct were analyzed (in online suppl. fig. 1) and it was found that maximal induction levels were achieved when 2CARD, MAVS200 and the chimera were coexpressed.

Recombinant Newcastle disease virus (NDV-GFP) has been shown to be a sensitive tool to detect IFN- $\beta$ [27]. We analyzed the capacity of the different molecules to prevent NDV-GFP replication. For this purpose, Vero cells were incubated for $24 \mathrm{~h}$ with supernatants of 293 cells previously transfected with MAVS, 2CARD and the 2CARD-MAVS200 plasmid. Vero cells were then infected with NDV-GFP, and GFP expression was analyzed under a fluorescent microscope (fig. 2d). A strong inhibition of GFP expression was observed in Vero cells pretreated with the supernatant obtained from the transfected cells. The amount of GFP in each well was quantified using ImageJ software and plotted. Analysis of the effective dose for each construct was calculated using GraphPad Prism 5. A higher dilution of the supernatant obtained from 2CARD-MAVS200 transfected cells was required to reach an effective inhibition of $50 \%$ of GFP expression (i.e. an effective concentration 50 or EC50), indicating the superior ability of this construct to create an antiviral status.

To assess the ability of 2CARD-MAVS200 to induce IFN- $\beta$ at the single-cell level, we utilized a reporter plasmid expressing GFP under the control of the IFN- $\beta$ promoter (pIFN- $\beta$-GFP). In order to normalize GFP expression, pIFN- $\beta$-GFP was cotransfected with a plasmid expressing iRFP protein under the control of the minimal cytomegalovirus promoter. We found that transfection of an empty plasmid or an iRFP-expressing plasmid had negligible IFN- $\beta$ induction effects (fig. 2e). Cells expressing this reporter system were cotransfected with a plasmid expressing RIG-I 2CARD, MAVS or the fusion 2CARD-MAVS200. Expression of all three constructs lead to induction of the IFN- $\beta$ promoter as determined by GFP expression. The percentage of IFN- $\beta$-GFP within the iRFP population was calculated using a GFP/iRFP ratio (fig. 2f). 2CARD-MAVS200 was able to induce IFN- $\beta$ more efficiently than 2CARD or MAVS alone in all IFN$\beta$-producing cells, not only in a particular cell subset (fig. $2 \mathrm{~g}$ ).

Next, we analyzed the role of endogenous MAVS in the capacity of the chimeric protein to induce the IFN- $\beta$ pathway. For this purpose, MAVS-deficient and WT control MEFs were transfected with a control plasmid or $2 \mathrm{CARD}$ or the chimeric protein-expressing plasmids, and IFN- $\beta$ mRNA expression was analyzed by quantitative RT-PCR. MAVS expression was absolutely required for IFN- $\beta$ induction by 2 CARD and 2CARD-MAVS200 (fig. 2h). To further characterize the contribution of MAVS to chimeric protein-mediated signaling, MAVS KO MEFs were cotransfected with the plasmid expressing the chimeric protein together with a plasmid expressing MAVS, and then compared to the ability of 2CARDMAVS200 to induce IFN- $\beta$. IFN- $\beta$ mRNA levels were significantly higher in the cells cotransfected with both plasmids than with the 2CARD-MAVS200 plasmid alone, indicating that MAVS expression is sufficient to rescue and enhance the IFN- $\beta$ stimulatory potential of 2 CARDMAVS200 (fig. 2i).

Human 2CARD, MAVS or 2CARD-MAVS Can

Induce IFN- $\beta$ in a Broad Spectrum of Cells from

Different Species

Once we had established the potential of the 2CARDMAVS200 construct to induce IFN- $\beta$ in 293 cells and their derivate $293 \mathrm{~T}$ cells, we next decided to explore the ability of this construct to induce IFN- $\beta$ in various cell lines from different organisms.

We performed IFN- $\beta$ reporter assays in adenocarcinoma human alveolar basal epithelial A549 cells, murine aneuploid fibrosarcoma L929 cells, SIRC cells, hamster pancreatic carcinoma-derived HaP-T1 cells and woodchuck hepatoma WCH-17 cells. In all cases, all the constructs showed the ability to induce IFN- $\beta$. Importantly, the human chimeric 2CARD-MAVS200 exhibited a significantly higher potential to induce the pathway when compared to RIG-I 2CARD or full-length MAVS in all cell lines (fig. 3).

\section{CARD-MAVS200 Induces the Transcription of}

Antiviral Genes in a Type I IFN-Independent Manner

Several antiviral genes do not require IFN signaling to be transcribed [8]. These genes have IRF-E DNA elements and are transcribed in response to IRF activation. Thus, signals that trigger IFN- $\beta$ induction and IRF3/IRF7 activation would induce transcription of antiviral genes (although this transcription can be further enhanced by type I IFN signaling).

In order to verify that $2 \mathrm{CARD}$, MAVS or 2CARDMAVS200 could act at the first stage of this process and induce antiviral genes in a type I IFN-independent way, we used STAT1 KO MEFs (fig. 4a). As a control, we used the corresponding WT MEFs. As expected, WT MEFs but not STAT1 KO MEFs responded to IFN- $\beta$ treatment by inducing transcription of the ISG genes tested. IFN- $\beta$ induction occurred when 2CARDMAVS200 was transfected into STAT1 KO cells as ex- 


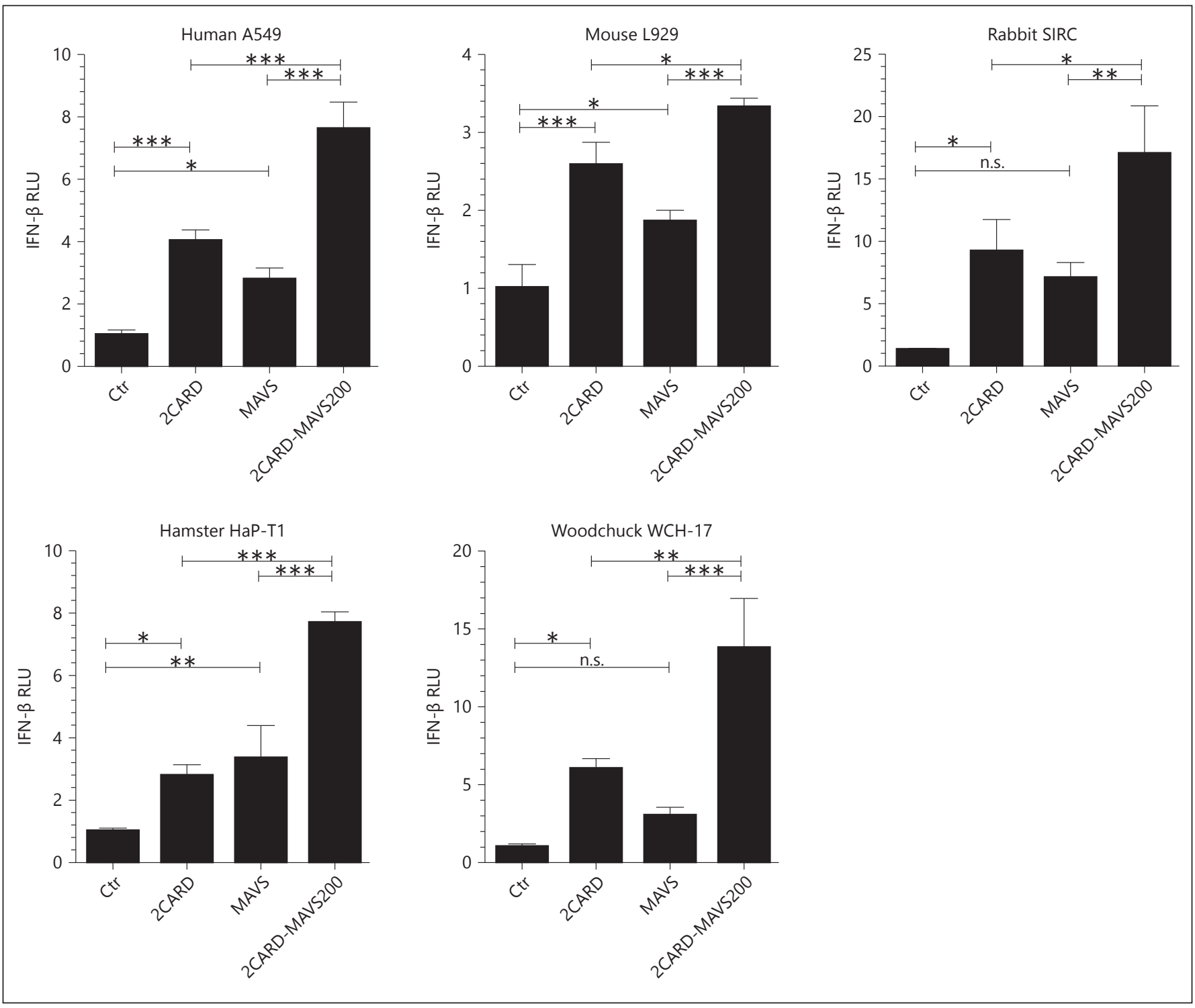

Fig. 3. Human RIG-I, MAVS and 2CARD-MAVS200 can activate the IFN- $\beta$ induction pathway in cells from multiple species. IFN- $\beta$ reporter assay in human A549 cells, mouse L929 cells, SIRC cells, HaP-T1 cells and WCH-17 cells. Cells were transfected with the corresponding reporter plasmids and $50 \mathrm{ng}$ of empty vector, 2CARD, MAVS or 2CARD-MAVS200. Ctr $=$ Control; n.s. $=$ not significant. ${ }^{*} \mathrm{p}<0.05,{ }^{* *} \mathrm{p}<0.01,{ }^{* * *} \mathrm{p}<0.001$.

of the type I IFN system were present. We chose three representative viral antagonist effectors, hepatitis $\mathrm{C}$ virus (HCV) NS3-4a protein, influenza virus NS1 protein and SV5 $\mathrm{V}$ protein.

HCV is known to block IFN- $\beta$ induction by the use of its NS3-4a protease, which cleaves MAVS [28]. In fact, Huh7.0 cells expressing the HCV genome [14] are deficient in their ability to induce IFN- $\beta$ in response to an RLR stimulus such as intracellular polyinosinic:polycytidylic acid (polyI:C) in comparison to Huh7.0 control 


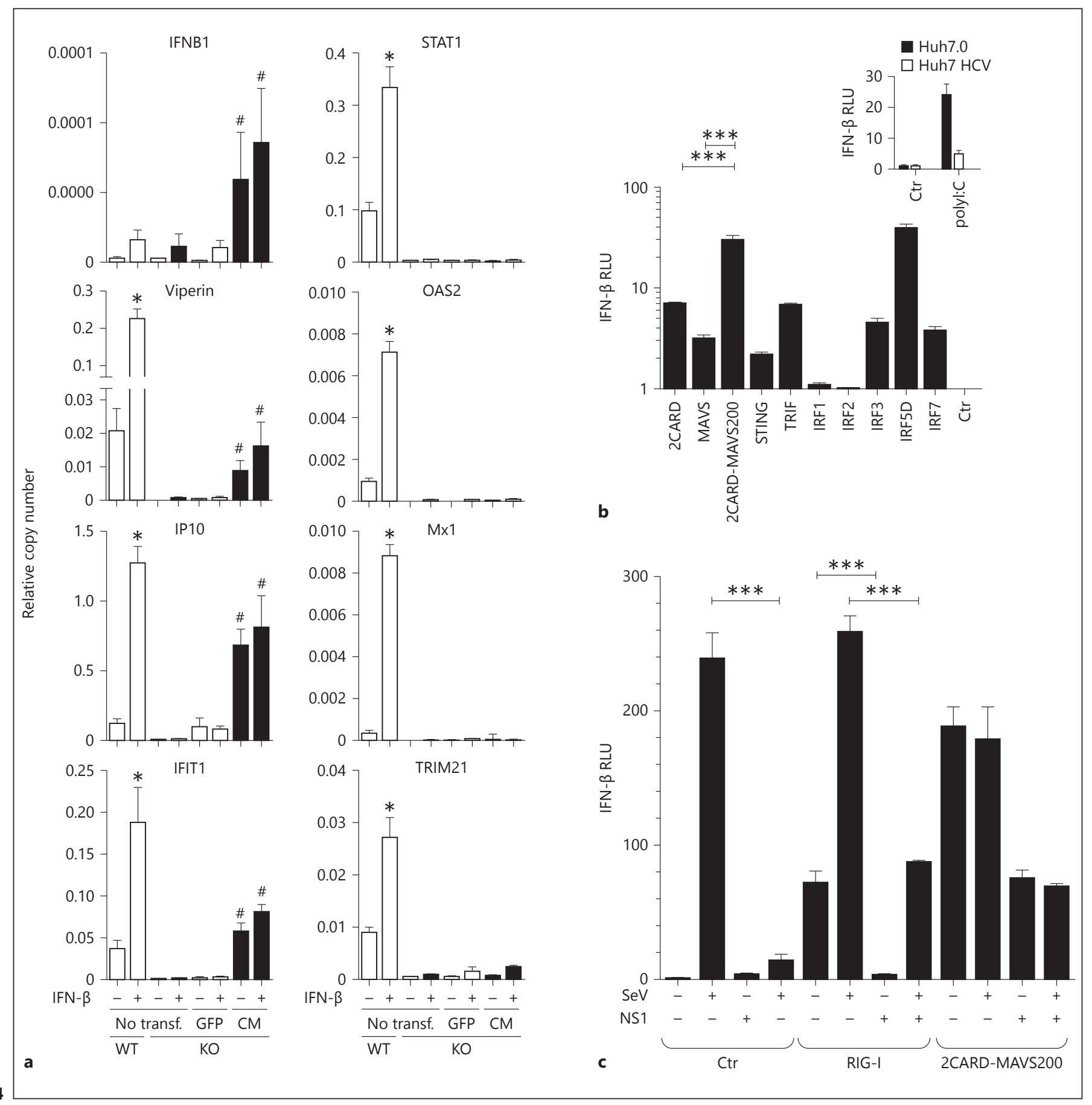

(For figure $d-f$ and legend see next page.)

parental cells (fig. $4 \mathrm{~b}$ ). We performed an IFN- $\beta$ reporter assay in Huh7.0 HCV cells overexpressing proteins that induce the IFN- $\beta$ pathway. Most of the proteins can overcome the antagonistic activity of the HCV NS3-4a, resulting in the induction of the pathway. Of all of them, phosphomimetic IRF-3 mutant (IRF3-5D), 2CARD-
MAVS200, 2CARD, TRIF, in this order, showed the greatest induction of the pathway. A residual IFN- $\beta$ induction was detected in polyI:C-transfected Huh7.0 HCV cells. Since MAVS is essential for IFN- $\beta$ induction through RLRs, we presume that the NS3-4a activity is not sufficient to completely abrogate MAVS-mediated signaling. 
Fig. 4. 2CARD-MAVS200 can partially recapitulate an antiviral program in IFN- $\beta$ induction or signaling scenarios. a Expression analysis of different genes involved in type I IFN activation between WT or STAT1 KO MEF cells, mock-treated or treated with mouse IFN- $\beta$ or STAT1 KO cells transfected with an empty vector or a plasmid expressing 2CARD-MAVS200. ${ }^{\#} \mathrm{p}<0.05,{ }^{*} \mathrm{p}<0.1$. b IFN $-\beta$ reporter assay of Huh7.0 cells constitutively expressing HCV nonstructural proteins. Cells were transfected with $1 \mu \mathrm{g}$ of the indicated constructs. c IFN- $\beta$ reporter assay of $293 \mathrm{~T}$ cells transfected with empty plasmids, RIG-I or 2CARD-MAVS200. These cells we cotransfected with a control plasmid or a plasmid expressing influenza PR8 NS1 protein. Cells were then subjected to mock or $\mathrm{SeV}$ infection. b, $\mathbf{c}^{* * *} \mathrm{p}<0.001$. d Gene expression analysis by qRT-PCR of IFN- $\beta$, MxA, ISG15 and OAS2. Parental 293 or stable 293 cells expressing SV5 V protein $(293 \mathrm{~V})$ were mock-treated or treated with $10^{4} \mathrm{IU}$ of human IFN- $\alpha 2$. In parallel, cells were transfected with $50 \mathrm{ng}$ of plasmids expressing GFP or 2CARD-MAVS200 for 24 h. ${ }^{*} \mathrm{p}<0.05$. e $293 \mathrm{~V}$ cells transfected with $500 \mathrm{ng}$ of an empty plasmid or plasmids expressing 2CARD, MAVS or 2CARD-MAVS200. Twenty-four hours after transfection, cells were infected with VSV (M51R)-GFP at an MOI of 0.001. Cellular supernatant was collected 24 h later. VSV titers in supernatant were quantified in Vero cells. As a control, cells were mocktreated or treated with $10^{4}$ IU of human IFN- $\alpha 2{ }^{*} \mathrm{p}<0.05,{ }^{* *} \mathrm{p}<0.01$. f VSV-GFP plaque size comparison of $293 \mathrm{~V}$-infected cells expressing 2CARD, MAVS, 2CARDMAVS200 or a control plasmid. CM = 2CARD-MAVS200; Ctr = control.
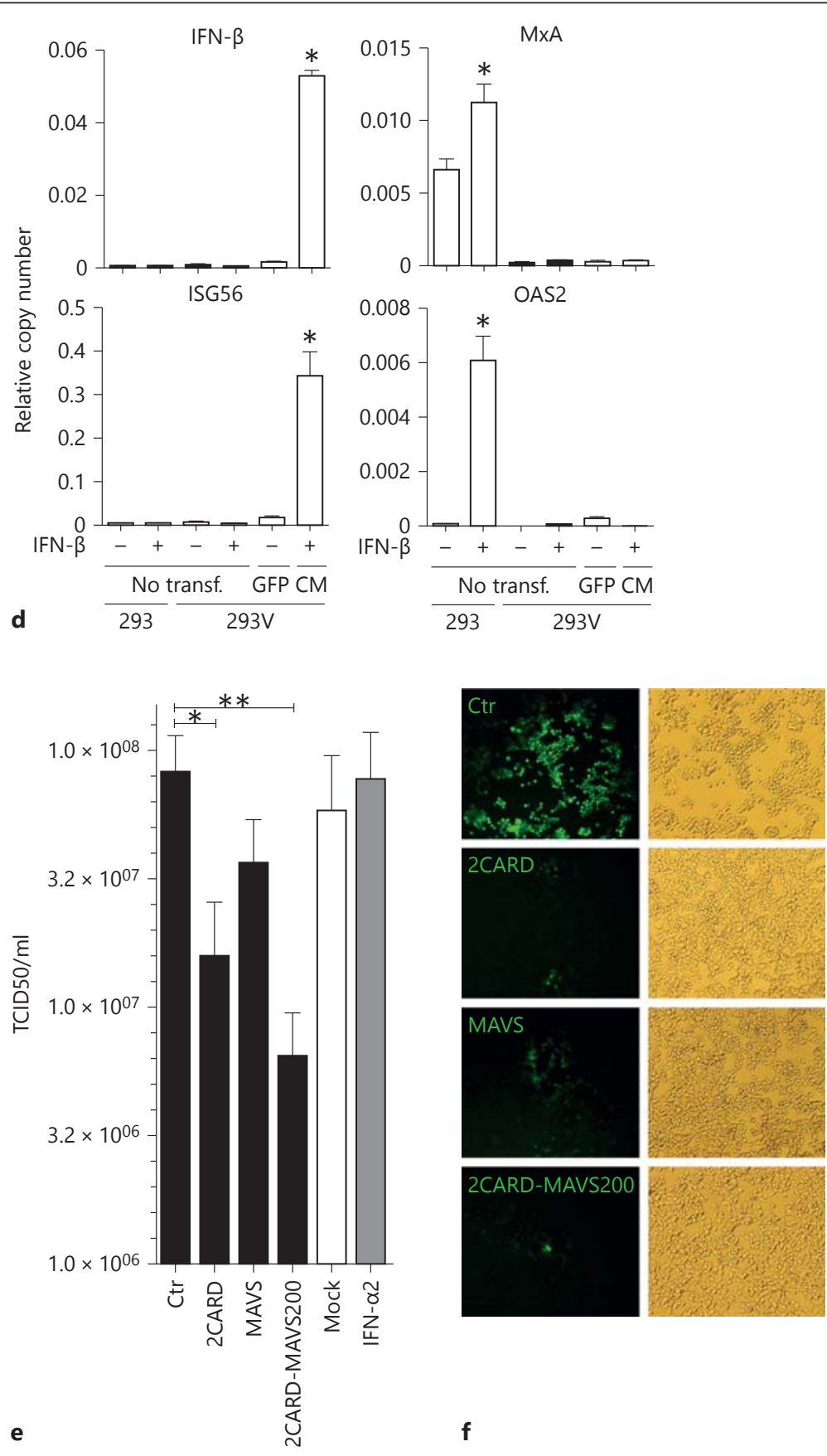

$\mathbf{f}$
Influenza $\mathrm{A} / \mathrm{PR} / 8 / 34$ virus NS1 protein has been widely described as a potent inhibitor of IFN- $\beta$ induction [35] through its ability to inhibit the RIG-I/MAVS interaction. We sought to determine whether the 2CARDMAVS200 construct has the ability to overcome the NS1 blockade of RIG-I-mediated signaling in response to $\mathrm{SeV}$ infection. NS1 expression clearly blocked the ability of
RIG-I overexpression to induce IFN- $\beta$ (approx. 2.4 times), while it only partially blocked (approx. 2.4 times) 2CARD-MAVS200-mediated IFN- $\beta$ induction (fig. 4c). Furthermore, as expected, NS1 overexpression blocked IFN- $\beta$ production induced by $\mathrm{SeV}$ infection. However, only a partial NS1-mediated inhibition was observed when cells overexpressing RIG-I (approx. 2.5 times) were 
infected with $\mathrm{SeV}$. SeV infection did not increase IFN- $\beta$ production in cells overexpressing 2CARD-MAVS200. However, 2CARD-MAVS200 was able to maintain the activation of the pathway in the presence of NS1, which only inhibited 2CARD-MAVS200 activity two times. SeV infection was unable to enhance the activation of the pathway in cells overexpressing NS1 plus 2CARDMAVS200, highlighting the ability of NS1 to inhibit endogenous RIG-I activation, but only partially inhibit 2CARD-MAVS activation.

Many viruses have developed potent antagonistic proteins to block type I IFN effects by hijacking or degrading elements of the type I IFN signaling cascade. SV5 V protein, for example, has been reported to bind and degrade STAT1 [29]. We hypothesized that RIG-I, MAVS or 2CARD-MAVS200 could partially recover the ability of these cells to create an antiviral state through the induction of antiviral genes, the transcription of which does not require type I IFN signaling. IFN- $\beta$ treatment of stable 293 cells expressing SV 5 V protein $(293 \mathrm{~V})$ failed to induce MxA and OAS2 expression in comparison to the parental 293 cells (fig. 4d). As expected, these cells could express IFN- $\beta$ and ISG56 genes in response to the transfection of a plasmid containing 2CARDMAVS200 but not type I IFN signaling-dependent genes such as MxA or OAS2, as we observed with STAT1 KO MEFs. Since some of the genes induced by 2CARDMAVS200 in 293V can act as direct antiviral genes, we explored whether this induction could be sufficient to partially block replication of a recombinant VSV-GFP virus. Cells were transfected with a control plasmid or plasmids expressing 2CARD, MAVS or 2CARDMAVS200. One day after transfection, cells were infected with rVSV-GFP at an MOI of 0.001. Twenty-four hours later, we took images of cells and titrated cell supernatants. Cells expressing 2CARD, MAVS or 2CARDMAVS200 could partially block VSV replication (fig. 4e, $\mathrm{f})$, highlighting the contribution made by IRF-E antiviral genes induced by the IFN- $\beta$ induction cascade to antiviral innate immunity.

Fig. 5. Gene transfer delivery of 2CARD-MAVS200 into lungs of mice using an AAV vector can protect mice from influenza virus infection. a Six-week old female C57BL/6 mice were intranasally inoculated with $1 \times 10^{11} \mathrm{vg} /$ mouse of $\mathrm{rAAV} 8$-EF1 viruses expressing luciferase, RIG-I 2CARD, MAVS or 2CARD-MAVS200 constructs and infected 3 days later with $10^{4} \mathrm{pfu} / \mathrm{mouse}$ of influenza $\mathrm{A} / \mathrm{PR} / 8 / 34$. Lung histology was analyzed 3 days after influenza virus infection by hematoxylin eosin staining (HE). b Influenza viral titers in lungs at days 3 and 5 after infection with the influenza
Gene Transfer Delivery of 2CARD-MAVS200 by Recombinant AAV Can Protect Mice from Influenza Virus Infection

Recombinant AAV vectors have been shown to be an effective method for delivering therapeutic genes in vivo. We developed three different AAVs expressing RIG-I 2CARD, MAVS or 2CARD-MAVS. Transgenes were subcloned into the AAV vector backbone under an elongation factor 1 promoter (EF1), and recombinant viruses were produced. We tested the antiviral activity of rAAV8EF1-RIG-I 2CARD, rAAV8-EF1-MAVS or rAAV8EF1-2CARD-MAVS200 in mice infected with influenza virus. As a control, we used rAAV8 virus expressing FF luciferase.

We first tested whether systemic administration of the different viruses had any effect on the animals. C57BL/6 mice $(n=6)$ were treated by intravenous injection with 3 doses of each virus, $5 \times 10^{9}, 1 \times 10^{10}$ or $5 \times 10^{10} \mathrm{vg} /$ mouse, rAAV8-EF1-2CARD, rAAV8-EF1-MAVS or rAAV8-EF1-2CARD-MAVS200 and AAV-EF-Luc was used as control. Type I IFN production and the potential hematological effect associated with it were analyzed in all the groups [30]. No IFN expression was detected in the serum of the different groups on days 3, 7 or 15 (data not shown). No sign of disease or hematologic toxicity was observed for the 2 months that the animals were monitored.

Next, we analyzed the antiviral effect of the vectors after intranasal administration. We administered the therapeutic vectors at a dose of $10^{11} \mathrm{vg} /$ mouse in a volume of $50 \mu \mathrm{l}$, using AAV8-FF-luciferase as a control and to monitor transgene expression. The expression of luciferase in the lungs of infected animals was monitored and a high level of expression was observed at day 3 (data not shown). As a result, mice were challenged with influenza virus at day 3 after AAV inoculation.

Animals were intranasally challenged with influenza $\mathrm{A} / \mathrm{PR} / 8 / 34$ at $10^{4} \mathrm{pfu} / \mathrm{animal}$ and sacrificed at days 3 and 5 after infection in order to analyze lung histology (fig. 5a; online suppl. fig. 2), viral titers (fig. 5b) and gene

virus. c Survival plot graph of animals infected with influenza: 6-week-old c57BL/6 females received $1 \times 10^{11} \mathrm{vg} /$ mouse of rAAV8-EF1-2CARD, MAVS or 2CARD-MAVS200 and 3 days later, were infected with $5 \times 10^{2} \mathrm{pfu} /$ animal of influenza $\mathrm{A} / \mathrm{PR} / 8 / 34$. qRT-PCR quantification of expression levels of influenza M1 gene (d), IFNB1 (e), Mx1 (f) and MHC-II (g) at days 3 and 5 after infection. ${ }^{*} \mathrm{p}<0.05,{ }^{* *} \mathrm{p}<0.01,{ }^{* * *} \mathrm{p}<0.001$, for all panels of the figure. $\mathrm{Ctr}=$ Control.

(For figure see next page.) 


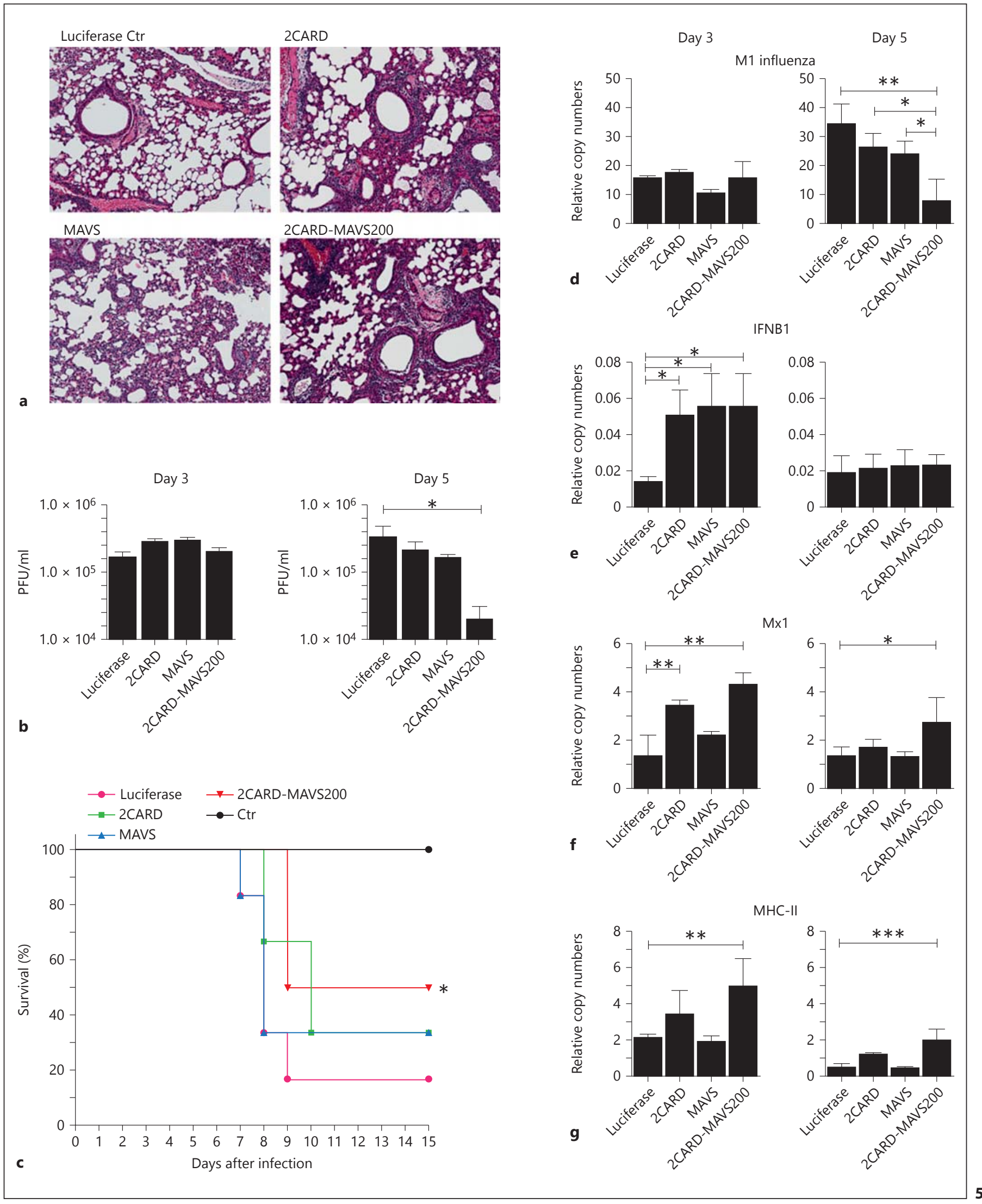

A RIG-I 2CARD-MAVS200 Chimera

J Innate Immun 2015;7:466-481 DOI: $10.1159 / 000375262$ 
expression (fig. 5d-g; online suppl. fig. 3). We observed moderate signs of infiltration at day 3 in the animals treated with AAV8-2CARD, and this was more marked in the AAV8-2CARD-MAVS200 group. Extensive infiltration was observed in all animals infected at day 5 but no differences among groups were detected at this time point (online suppl. fig. 2). Influenza virus replication was significantly inhibited in mice transduced with AAV-EF1-2CARD-MAVS200 in comparison to the control group. At day 5, viral titers were one log lower in the 2CARD-MAVS200-treated group than in control animals. No difference between groups was observed at day 3 (fig. 5b).

The treatment effect on animal survival was also analyzed. Animals received an intranasal dose of $10^{11} \mathrm{vg}$ of the corresponding AAV. Three days later, the mice were challenged with influenza at a dose of $5 \times 10^{2} \mathrm{pfu} / \mathrm{animal}$ and the survival of the animals was monitored every day for 2 weeks (fig. 5c). Animals treated with AAV8-2CARDMAVS200 presented a significant difference in survival from the mice that received AAV8-FF-luciferase. Mice that received RIG-I 2CARD or MAVS showed a small but no significant effect in survival when compared to the control group.

The levels of viral mRNA were measured by qRT-PCR specific for influenza M1 gene in the lungs of the infected animals. No significant differences were observed at day 3 , but at day 5 , in correlation with viral titers, viral gene expression was lower in the animals treated with the chimera (fig. $5 d$ ). A significant increase in IFN- $\beta$ mRNA expression was detected in animals treated with the different constructs at day 3 but no difference was observed at day 5 (fig. 5e). We then examined the type I IFN signature by analyzing $\mathrm{Mxl}$ and MHC-II expression (fig. 5f, g). Expression of these genes was significantly higher at day 5 in the animals treated with AAV expressing the chimera in comparison to the other groups, correlating with a stronger antiviral effect. No significant differences in the expression of IRF-E genes such as viperin, IFIT1, IFNL or IP10 were observed. A small but significant increase in TNF- $\alpha$ expression was observed at days 3 and 5 in the animals treated with AAV-2CARD-MAVS200 (online suppl. fig. 3)

Gene Transfer Delivery of 2CARD-MAVS200 by Recombinant $A A V$ Can Interfere with Hepatitis $B$ Replication in Transgenic HBV Mice

Transgenic HBV1.3 (HBV Tg) mice have been used to test new antiviral therapies against HBV chronic infection [31]. Effective therapies are associated with reduced viremia or reduced detection of viral antigens such as core protein $(\mathrm{HBcAg})$. We aimed to study whether 2CARD-MAVS-200 could influence HBV replication in these animals.

AAV8-EF1-2CARD-MAVS200 was intravenously injected in HBV Tg mice and animals were monitored for HBV genome levels in the blood. We detected a 10 -fold reduction in the amount of $\mathrm{HBV}$ genomes as early as 2 days after administration of $5 \times 10^{10} \mathrm{vg} /$ mouse of AAV8-2CARD-MAVS200 (fig. 6a). However, this initial reduction had disappeared by day 7. In order to confirm that the effect of this drop at day 2 was due to an antiviral effect in mouse hepatocytes, we analyzed levels of $\mathrm{HBcAg}$ in the livers of the treated animals by immunohistochemistry. Mice received a dose of AAV as previously described and were sacrificed 2 days later. Animals treated with AAV8-2CARD-MAVS presented a significant reduction in the amount of cytoplasmic core protein in hepatocytes compared to a control group treated with AAV8-2CARDluciferase or untreated mice. Expression of the transgenes was verified by qRT-PCR (fig. 6b).

Liver extracts and serum samples were analyzed in an attempt to detect IFN- $\beta$, but we failed to see protein expression at this time point. Furthermore, IFN- $\beta$ and IFN- $\lambda$ mRNA could not be detected in the liver by qRTPCR analysis, and IFN- $\alpha$ expression was very low and remained unchanged after the treatment (online suppl. fig. 4). However, an increase in the expression of IFNstimulated genes such as Mx1 (fig. 6c) or MHC-II (fig. 6d) could be detected in the liver extract 2 days after vector administration. Expression of 2CARD-MAVS200 (fig. 6e) and luciferase (fig. 6f) was confirmed by qRTPCR in the liver of AAV-treated animals.

\section{Discussion}

Viruses employ a multitude of strategies to avoid the induction of type I IFN and to evade its antiviral effects [11]. Many studies have identified and described these viral antagonistic mechanisms and many efforts have been oriented towards the development of drugs to overcome the inhibitory effects. Here, we propose a new strategy based on the expression of a chimeric protein 2CARDMAVS200 (2CARD domain of RIG-I fused to a downstream interacting protein fragment, the first 200 amino acids of the protein MAVS).

To our knowledge, the 2CARD-MAVS200 chimera is the first artificial construct with an increased ability to induce IFN- $\beta$ as compared to the corresponding in- 


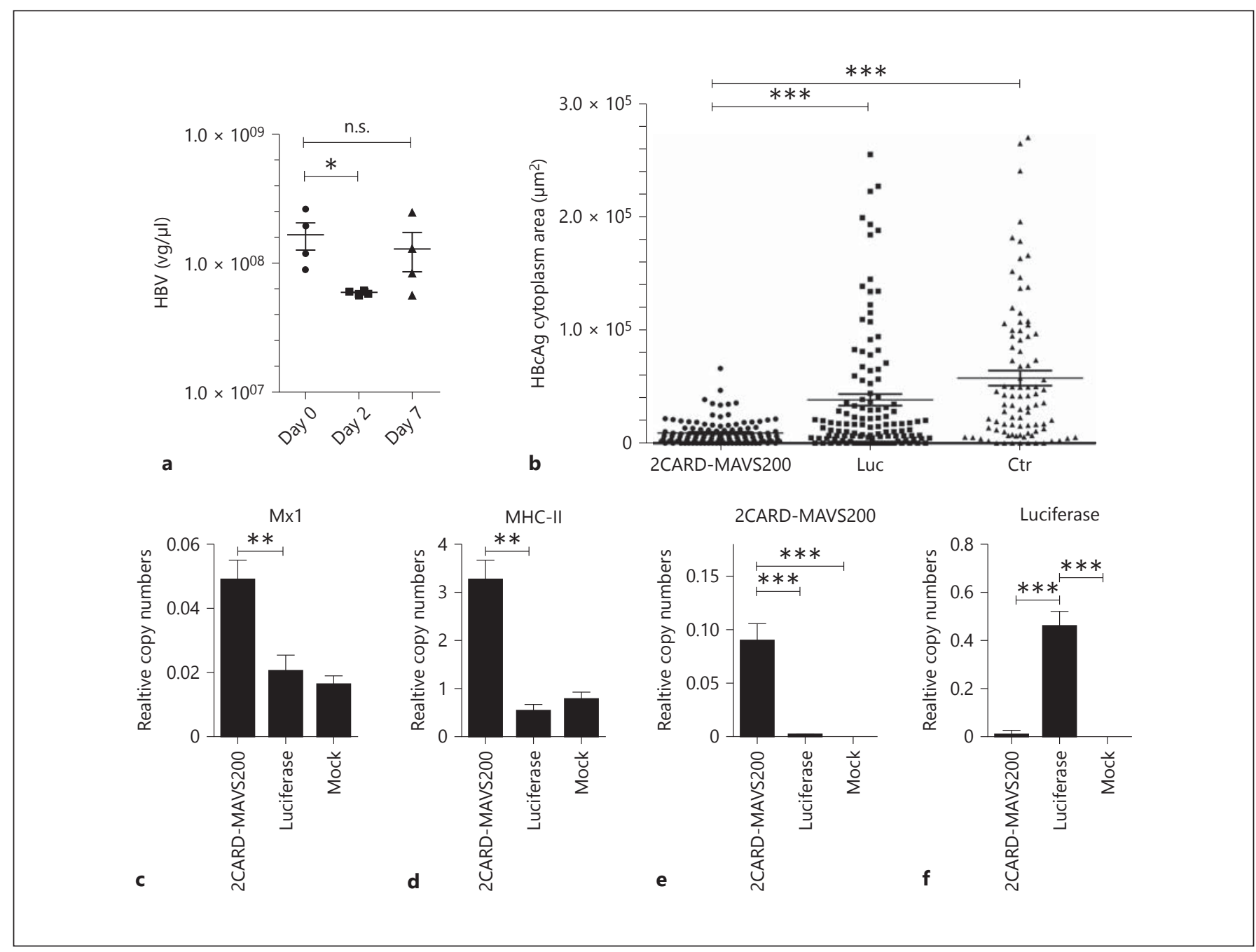

Fig. 6. rAAV-EF1-2CARD-MAVS200 treatment in HBV transgenic mice can interfere with virus replication at early time points. a Ten-week-old female transgenic HBV mice were treated intravenously with $5 \times 10^{10} \mathrm{vg} /$ animal of rAAV8-EF1-2CARD-MAVS200. $\mathrm{HBV}$ replication was monitored by the presence of $\mathrm{vg}$ in the blood by qPCR. ${ }^{*} \mathrm{p}<0.05$. $\mathbf{b}$ Quantification of the cytoplasmic area positive to HBcAg (detected by immunohistochemistry) in hepato-

dividual chimeric components of the RIG-I signaling pathway. Although other chimeric constructs that enhance type I IFN signaling have been tested [32], this is the first chimera that can stimulate the IFN- $\beta$ induction pathway, mimicking RLR-dependent induction. This chimeric construct fuses two domains that normally interact during activation of the IFN- $\beta$ pathway. MAVS200 contains a CARD domain and a PRR domain. MAVS CARD has two polar surfaces which are predicted to mediate homotypic CARD:CARD protein interaction [33]. This domain has been proven as necessary and suf- cytes of 2CARD-MAVS200-treated mice or the corresponding control groups 2 days after AAV treatment. ${ }^{* * *} \mathrm{p}<0.001$. Induction of Mxl (c) and MHC-II (d) gene expression quantified by qRT-PCR. ${ }^{* *} \mathrm{p}<0.01$. Transgene expression levels of 2CARDMAVS (e) or luciferase (f) quantified by qRT-PCR in the liver of the animals transduced with the corresponding AAV vectors. ${ }^{* * *} \mathrm{p}<0.001$. Ctr $=$ Control; $\mathrm{n} . \mathrm{s} .=$ not significant.

ficient to bind RIG-I 2CARD, and also to trigger the formation of prion-like structures, the formation of which seems to be important for the optimal activation of the pathway $[34,35]$. The PRR domain is a prolineenriched section with several consensus binding sites for proteins of the TRAF family. TRAF2, TRAF3 and TRAF6 have been shown to bind to MAVS within this PRR domain [6, 36]. Overexpressing 2CARD-MAVS200 can bypass at least two required steps for the activation of IFN- $\beta$ expression. First, expression of $2 \mathrm{CARD}-$ MAVS200 introduces RIG-I 2CARD in an already ac- 
tive conformation, bypassing the requirement for RIGI conformational change, second, it is presented already bound to MAVS2000, and finally, overexpression of MAVS200 may contribute to formation of MAVS prion-like structures together with endogenous MAVS. This increased efficiency of signaling events may explain the higher percentage of cells inducing IFN- $\beta$ (fig. $2 \mathrm{e}-\mathrm{g}$ ). We also found that the mechanism to induce IFN- $\beta$ induction pathway by the chimera is dependent on full-length MAVS, which is necessary (fig. $2 \mathrm{~h}$ ) and sufficient (fig. 2i) to achieve the properties of 2 CARDMAVS200.

Furthermore, human RIG-I 2CARD, MAVS or 2CARD-MAVS200 seem to retain a pan-specific ability to trigger IFN- $\beta$ signaling in different species. This behavior points to a conserved activation mechanism, which may not require species-specific regulatory events.

Interestingly, in the absence of type I IFN signaling, 2CARD-MAVS200 can create an antiviral state. As we show here, the antiviral proteins viperin [37] and IFIT1 [38] are induced by the chimera in the absence of type I IFN signaling (fig. 4). Other genes induced by $2 \mathrm{CARD}$ MAVS200, independent of type I IFN signaling, are potent inducers of the immune system, such as the chemokine IP10/CXCL10 [39]. Induction of these genes as well as the lack of induction of many other ISG indicates, however, that an IFN signaling antiviral program is required for a full antiviral response.

This construct has several interesting properties, including the induction of high levels of IFN- $\beta$ and the ability to stimulate the IFN- $\beta$ pathway in a high percentage of cells even in the absence of external stimuli. This chimera also exhibits a superior ability to overcome (at least in part) viral restrictions mediated by $\mathrm{HCV}$ proteins, influenza virus NS1 protein and SV5 V proteins in comparison to other inducers. Furthermore, 2CARDMAVS200 was delivered into mice using a recombinant $A A V$ vector and its therapeutic activity was tested against influenza virus and HBV.

Several diseases including cancers and chronic viral infections can be treated with recombinant type I IFN [25]. In some cases, this treatment is ineffective, in part, due to the refractory response by the tumor or the infected tissue. This lack of response can be attributed to mutations in genes that control the pathway or disruption of type I IFN signaling by viral antagonistic elements. Our results show that overexpression of 2CARDMAVS200 may activate a program that recapitulates, at least in part, some of the effects induced by type I IFN signaling.
We explored the possibility of using rAAV expressing 2CARD, MAVS or 2CARD-MAVS200 in vivo in acute and chronic viral infection mouse models. Constitutive expression of 2CARD, MAVS or 2CARD-MAVS200 under the transcriptional control of the elongation factor $1 \alpha$ promoter (EF1) resulted in an efficient but modest activation of an antiviral program. No expression of IFN- $\beta$ could be detected in the blood after 2 days, although the signature of type I signaling was shown by the detection of the expression of ISG genes inside the transduced organs. The transient nature of antiviral signaling induced by 2CARD, MAVS or 2CARD-MAVS200 could be explained by the activation of various negative regulatory mechanisms known to turn off IFN signaling [22, 40].

An alternative strategy which may bypass the shut-off of antiviral signaling could involve transient expression of the transgene under the control of an inducible promoter. Deregulation of repressive mechanisms could also potentiate the length or strength of the response. Phosphorylation of RIG-I 2CARD is used by the cell to maintain steady-state inactivated RIG-I. Mutation of phosphorylation sites of RIG-I 2CARD to alanine have been shown to bypass the negative regulatory mechanisms.

We hypothesize that 2CARD-MAVS200 functions as an inducer of endogenous MAVS signaling platforms at the surface of the mitochondria, recapitulating RIG-I 2CARD:MAVS interaction and activation. This tool can be used to reconstitute defective IFN- $\beta$ activation in pathogenic scenarios. Further studies are needed to characterize the specific immunostimulatory properties of this chimeric protein.

\section{Acknowledgements}

We thank Richard Cadagan (Icahn School of Medicine at Mount Sinai) for his invaluable and enduring technical support. Thanks to Lisa Miorin, Mirco Schmolke, Armando José Moreno Cermeño and Alina Baum for valuable reagents and suggestions. This study was partly supported by CRIP (Center for Research on Influenza Pathogenesis), an NIAID funded Center of Excellence for Influenza Research and Surveillance (CEIRS, contract number HHSN272201400008C) to AG-S, and by the Spanish MINECO Fellowship JCI-2011-09179 and SAF2012-39578, by the ISCIII, cofounded by FEDER, grant No. PI11/01534 and the European Marie-Curie IRG-2010-277172 to EN-V.

\section{Disclosure Statement}

The authors have no conflicts of interest to declare. 


\section{References}

$\checkmark 1$ Broz P, Monack DM: Newly described pattern recognition receptors team up against intracellular pathogens. Nat Rev Immunol 2013;13:551-565.

2 Gonzalez-Navajas JM, Lee J, David M, Raz E: Immunomodulatory functions of type I interferons. Nat Rev Immunol 2012;12:125-135.

$\checkmark 3$ Medzhitov R: Approaching the asymptote: 20 years later. Immunity 2009;30:766-775.

-4 Loo YM, Gale M Jr: Immune signaling by RIGI-like receptors. Immunity 2011;34:680-692.

5 Panne D: The enhanceosome. Curr Opin Struct Biol 2008;18:236-242.

6 Xu LG, Wang YY, Han KJ, Li LY, Zhai Z, Shu HB: VISA is an adapter protein required for virus-triggered IFN-beta signaling. Mol Cell 2005; 19:727-740.

7 Watanabe N, Sakakibara J, Hovanessian AG, Taniguchi T, Fujita T: Activation of IFN-beta element by IRF-1 requires a posttranslational event in addition to IRF-1 synthesis. Nucleic Acids Res 1991;19:4421-4428.

-8 Grandvaux N, Servant MJ, tenOever B, Sen GC, Balachandran S, Barber GN, Lin R, Hiscott J: Transcriptional profiling of interferon regulatory factor 3 target genes: direct involvement in the regulation of interferonstimulated genes. J Virol 2002;76:5532-5539.

$>9$ Wu C, Ohmori Y, Bandyopadhyay S, Sen G, Hamilton T: Interferon-stimulated response element and NF kappa B sites cooperate to regulate double-stranded RNA-induced transcription of the IP-10 gene. J Interferon Res 1994; 14:357-363.

10 Ivashkiv LB, Donlin LT: Regulation of type I interferon responses. Nat Rev Immunol 2014; 14:36-49.

$>11$ Versteeg GA, Garcia-Sastre A: Viral tricks to grid-lock the type I interferon system. Curr Opin Microbiol 2010;13:508-516.

-12 Zhao M, Zhang J, Phatnani H, Scheu S, Maniatis T: Stochastic expression of the interferon-beta gene. PLoS Biol 2012;10:e1001249.

-13 Tunon MJ, San Miguel B, Crespo I, Riezu-Boj JI, Larrea E, Alvarez M, Gonzalez I, Bustos M, Gonzalez-Gallego J, Prieto J: Cardiotrophin-1 promotes a high survival rate in rabbits with lethal fulminant hepatitis of viral origin. J Virol 2011;85:13124-13132.

14 Larrea E, Riezu-Boj JI, Aldabe R, Guembe L, Echeverria I, Balasiddaiah A, Gastaminza P, Civeira MP, Sarobe P, Prieto J: Dysregulation of interferon regulatory factors impairs the expression of immunostimulatory molecules in hepatitis $\mathrm{C}$ virus genotype 1-infected hepatocytes. Gut 2014;63:665-673.

15 Garcia-Sastre A, Durbin RK, Zheng H, Palese P, Gertner R, Levy DE, Durbin JE: The role of interferon in influenza virus tissue tropism. J Virol 1998;72:8550-8558.

-16 Kumar H, Kawai T, Kato H, Sato S, Takahashi K, Coban C, Yamamoto M, Uematsu S, Ishii KJ, Takeuchi O, Akira S: Essential role of IPS1 in innate immune responses against RNA viruses. J Exp Med 2006;203:1795-1803.
17 Hai R, Martinez-Sobrido L, Fraser KA, Ayllon J, Garcia-Sastre A, Palese P: Influenza B virus NS1-truncated mutants: live-attenuated vaccine approach. J Virol 2008;82:1058010590.

18 Mibayashi M, Martinez-Sobrido L, Loo YM, Cardenas WB, Gale M Jr, Garcia-Sastre A: Inhibition of retinoic acid-inducible gene I-mediated induction of beta interferon by the NS1 protein of influenza A virus. J Virol 2007;81: 514-524.

19 Gack MU, Albrecht RA, Urano T, Inn KS, Huang IC, Carnero E, Farzan M, Inoue S, Jung JU, Garcia-Sastre A: Influenza A virus NS1 targets the ubiquitin ligase TRIM25 to evade recognition by the host viral RNA sensor RIG-I. Cell Host Microbe 2009;5:439_ 449.

20 Reed LJ, Muench H: A simple method of estimating fifty per cent endpoint. Am J Hyg 1938;27:493-497

21 Park MS, Steel J, Garcia-Sastre A, Swayne D, Palese P: Engineered viral vaccine constructs with dual specificity: avian influenza and Newcastle disease. Proc Natl Acad Sci U S A 2006;103:8203-8208.

22 Nistal-Villan E, Gack MU, Martinez-Delgado G, Maharaj NP, Inn KS, Yang H, Wang R, Aggarwal AK, Jung JU, Garcia-Sastre A: Negative role of RIG-I serine 8 phosphorylation in the regulation of interferon-beta production. J Biol Chem 2010;285:20252-20261.

23 Berraondo P, Ochoa L, Crettaz J, Rotellar F, Vales A, Martinez-Anso E, Zaratiegui M, Ruiz J, Gonzalez-Aseguinolaza G, Prieto J: IFN-alpha gene therapy for Woodchuck hepatitis with adeno-associated virus: differences in duration of gene expression and antiviral activity using intraportal or intramuscular routes. Mol Ther 2005;12:68-76.

24 Abramoff MDM, Paulo J, Ram SJ: Image processing with ImageJ. Biophotonics International 2004; 11:36-42.

25 George PM, Badiger R, Alazawi W, Foster GR, Mitchell JA: Pharmacology and therapeutic potential of interferons. Pharmacol Ther 2012;135:44-53.

26 Baum A, Sachidanandam R, Garcia-Sastre A: Preference of RIG-I for short viral RNA molecules in infected cells revealed by next-generation sequencing. Proc Natl Acad Sci U S A 2010;107:16303-16308.

27 Park MS, Shaw ML, Munoz-Jordan J, Cros JF, Nakaya T, Bouvier N, Palese P, Garcia-Sastre A, Basler CF: Newcastle disease virus (NDV)based assay demonstrates interferon-antagonist activity for the NDV $\mathrm{V}$ protein and the Nipah virus $V, W$, and $C$ proteins. J Virol 2003;77:1501-1511.
28 Li XD, Sun L, Seth RB, Pineda G, Chen ZJ: Hepatitis C virus protease NS3/4A cleaves mitochondrial antiviral signaling protein off the mitochondria to evade innate immunity. Proc Natl Acad Sci U S A 2005;102:17717-17722.

29 Didcock L, Young DF, Goodbourn S, Randall RE: The V protein of simian virus 5 inhibits interferon signalling by targeting Stat1 for proteasome-mediated degradation. J Virol 1999;73:9928-9933.

30 Kirkwood JM, Bender C, Agarwala S, Tarhini A, Shipe-Spotloe J, Smelko B, Donnelly S, Stover L: Mechanisms and management of toxicities associated with high-dose interferon alpha-2b therapy. J Clin Oncol 2002;20:37033718.

31 Cavanaugh VJ, Guidotti LG, Chisari FV: Interleukin-12 inhibits hepatitis B virus replication in transgenic mice. J Virol 1997;71:3236-3243.

$>32$ Kraus TA, Lau JF, Parisien JP, Horvath CM: A hybrid IRF9-Stat2 protein recapitulates interferon-stimulated gene expression and antiviral response. J Biol Chem 2003;278:1303313038.

33 Potter JA, Randall RE, Taylor GL: Crystal structure of human IPS-1/MAVS/VISA/Cardif caspase activation recruitment domain. BMC Struct Biol 2008;8:11.

34 Hou F, Sun L, Zheng H, Skaug B, Jiang QX, Chen ZJ: Mavs forms functional prion-like aggregates to activate and propagate antiviral innate immune response. Cell 2011;146:448461 .

35 Cai X, Chen J, Xu H, Liu S, Jiang QX, Halfmann R, Chen ZJ: Prion-like polymerization underlies signal transduction in antiviral immune defense and inflammasome activation. Cell 2014;156:1207-1222.

36 Saha SK, Pietras EM, He JQ, Kang JR, Liu SY, Oganesyan G, Shahangian A, Zarnegar B, Shiba TL, Wang Y, Cheng G: Regulation of antiviral responses by a direct and specific interaction between TRAF3 and Cardif. EMBO J 2006;25:3257-3263.

37 Chin KC, Cresswell P: Viperin (CIG5), an IFN-inducible antiviral protein directly induced by human cytomegalovirus. Proc Natl Acad Sci U S A 2001;98:15125-15130.

38 Pichlmair A, Lassnig C, Eberle CA, Gorna MW, Baumann CL, Burkard TR, Burckstummer T, Stefanovic A, Krieger S, Bennett KL, Rulicke T, Weber F, Colinge J, Muller M, Superti-Furga G: IFIT1 is an antiviral protein that recognizes $5^{\prime}$-triphosphate RNA. Nat Immunol 2011;12:624-630.

39 Dufour JH, Dziejman M, Liu MT, Leung JH, Lane TE, Luster AD: IFN-gamma-inducible protein 10 (IP-10; CXCL10)-deficient mice reveal a role for IP-10 in effector T cell generation and trafficking. J Immunol 2002;168: 3195-3204.

40 Dalpke A, Heeg K, Bartz H, Baetz A: Regulation of innate immunity by suppressor of cytokine signaling (SOCS) proteins. Immunobiology 2008;213:225-235.
A RIG-I 2CARD-MAVS200 Chimera

Restores Type I IFN Response
J Innate Immun 2015;7:466-481 DOI: $10.1159 / 000375262$ 